\title{
Responsabilidad social de las empresas y beneficios privados: el impacto de la orientación estratégica en empresas grandes ubicadas en México
}

\section{Corporative social responsibility and private benefits: the impact of strategic orientation in large companies in Mexico}

\author{
JosÉ DE Jesús SAlaZAR-CANTÚ* \\ EsTHELA GUTIÉRREZ-GARZA** \\ KARIM ACUÑA-ASKAR** \\ Bryan William HuSTED-CORREGAN*
}

\begin{abstract}
In this article we analyse new theoretical proposals about three main lines regarding the expenditure on corporative social responsibility (CSR) and construct indices by type of expense in CSR, as well as a competitiveness index. In order to do this, we have employed regression models to find what kind of expense in CSR directly affects the expected return. The new theoretical proposal puts into question a great deal of the empirical evidence of the relationship between CSR expenditure and the profitability of the companies that apply it.
\end{abstract}

Keywords: strategy, corporative social responsibility, competitiveness.

\section{Resumen}

En este artículo se analizan nuevas propuestas teóricas sobre tres orientaciones principales en el gasto en responsabilidad social corporativa (RSC) y se construyen índices por tipo de gasto en RSC, así como un índice de competencia. Para ello se emplearon modelos de regresión y se encontró que el tipo de gasto en RSC influye directamente sobre la utilidad esperada. La nueva propuesta teórica cuestiona gran parte de la evidencia empírica sobre la relación que guardan los gastos en RSC y la rentabilidad de las empresas que los llevan a cabo.

Palabras clave: estrategia, responsabilidad social corporativa, competencia.

* Instituto Tecnológico y de Estudios Superiores de Monterrey, Campus Monterrey. Correos-e: jsalazar@itesm.mx, bhusted@itesm.mx

** Universidad Autónoma de Nuevo León. Correos-e: egutierr@ccr.dsi.uanl.mx, karaskar@yahoo.com,kaskar@fm.uanl.mx 


\section{Introducción ${ }^{1}$}

Las acciones de las empresas en lo que se denomina RSC son una forma de reducir la tensión entre los objetivos sociales y los de utilidad privada, la empresa asigna recursos que permiten alcanzar objetivos sociales de largo plazo al tiempo que crea valor para los accionistas.

Se han dedicado muchas investigaciones a examinar la relación entre los desempeños económico y social de las empresas (Griffin y Mahon, 1997; Waddock y Graves, 1997; McWilliams y Siegel, 2000; Margolis y Walsh, 2001). Hasta ahora los resultados son mixtos: algunos estudios muestran una relación positiva entre estos dos aspectos, en otros, es al contrario y en otros más no se encuentra relación significativa.

"La evidencia sobre este tipo de relaciones y de las implicaciones sociales de esta forma de participación de la empresa, es incipiente" (Baron, 2001: 42). México es un interesante caso de estudio en el campo de la RSC tanto porque es un país en desarrollo, como por lo incipiente de este tipo de investigaciones en Latinoamérica. A este respecto, Estrella Peinado señala:

La información disponible sobre la implementación de responsabilidad social corporativa en Latinoamérica es contradictoria y mucha de la evidencia es circunstancial. La actividad de responsabilidad social corporativa por lo pronto se ve relativamente débil y desigualmente distribuida, con muy pocas compañías con una agenda formal de responsabilidad social corporativa que asegure condiciones laborales saludables y comunidades habitables (2004: 7).

Igualmente, María Correa et al.(2004) al referirse al estudio de este concepto en Latinoamérica, dan una idea de lo nuevo que es el tratamiento del tema en estas latitudes: “...la concepción de la responsabilidad social de la empresa como parte de la gestión de negocios y dentro de un concepto integral es un tema con casi 10 años de presencia en la región” (2004: 7). También señalan que todavía se nota la fuerte presencia de la filantropía como orientación principal detrás del ejercicio de responsabilidad social empresarial.

\footnotetext{
${ }^{1}$ Este estudio forma parte del capítulo $\mathrm{v}$ de la tesis realizada por José de Jesús Salazar Cantú, dentro del programa de doctorado en ciencias sociales con orientación en desarrollo sustentable, del Instituto de Investigaciones Sociales de la Universidad Autónoma de Nuevo León.
} 
Para el caso de México, María Lara (2000: 5) señala que entre las empresas grandes, $88 \%$ hace donativos a causas sociales diversas y $12 \%$ no, también indica algunas de las razones para otorgarlos, en orden de importancia éstas son: responsabilidad, generosidad, incentivo fiscal e imagen, estos dos últimos empatados. También para México, Sabrina Salazar asevera que "Existen muy pocas investigaciones sobre el estudio de la evolución de la responsabilidad social corporativa en México y de sus efectos" (2004: 3). Hasta ahora, no se han hecho estudios empíricos para México que relacionen la orientación preponderante del ejercicio de responsabilidad social y su relación con el desempeño financiero y el nivel de competencia ${ }^{2}$ de la empresa, esta relación se estudia en el presente trabajo.

El estudio contempla dos nuevas orientaciones en la relación entre las inversiones en responsabilidad social y el desempeño financiero de la empresa. Primero se parte de la tipificación del sesgo en el gasto en RSC propuesta por Bryan Husted y José Salazar (2006), de acuerdo con la cual se esperaría una relación diferente entre rentabilidad y ejercicio de responsabilidad social, según el sesgo de este último hacia lo estratégico, lo altruista o lo

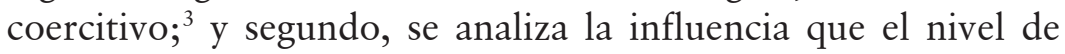
competencia puede tener en el efecto esperado sobre la utilidad de la empresa derivado de su gasto en RSC, esta segunda orientación tiene como base los planteamientos teóricos propuestos en Salazar y Husted ( 2005). En la sección de marco teórico se amplía la explicación de estas nuevas propuestas teóricas en el campo de la RSC.

Dada la magnitud de los problemas que enfrenta la población, las empresas mexicanas experimentan una fuerte presión por ayudar en lo social; quizá por ello, los asuntos de ética de los negocios y la responsabilidad social cada vez están más presentes en el medio y foros relevantes de los negocios en México (Cemefi, 2004). No se trata sólo de una demanda social, sino de algo que los propios consumidores aquilatan, así lo señalan Timothy Feddersen y Thomas Guilligan: "En las economías desarrolladas, los patrones de compra entre los consumidores trascienden la simple economía del valor relativo e incluyen apreciaciones morales y políticas" (2001: 149). Para el caso del consumidor mexicano, este aspecto lo confirman Valdés et al.

\footnotetext{
${ }^{2}$ Cuando aquí hablamos de competencia aludimos al nivel de rivalidad que prevalece en el mercado principal en el que participa la empresa.

${ }^{3}$ En el anexo se presentan los modelos empleados por estos autores.
} 
(1999: 177), donde se encuentra que 74\% de los consumidores estarían dispuestos a comprar preferentemente productos que apoyen causas sociales. Estos elementos vienen a dar mayor justificación a la realización de estudios en el campo de la RSC.

Los resultados más importantes del presente artículo permiten observar de manera exploratoria que entre el grupo de empresas que componen el estudio, el gasto en RSC no está asociado con la obtención de mayores niveles de rentabilidad. Al distinguir la motivación de dicho gasto como variable explicativa, el sesgo estratégico lleva a una mayor rentabilidad, mientras el coercitivo tiene que ver con menores niveles de ésta y el altruista no presenta asociación estadística alguna. Finalmente, también se muestra que altos niveles de competencia se relacionan más con la existencia de gastos en RSC de corte coercitivo y estratégico, pero no con los de sesgo altruista.

\section{Marco teórico}

\subsection{Sobre la relación entre gasto en $\mathrm{RSC}$, utilidad de la empresa y producto social}

El estudio toma como base de análisis dos nuevas propuestas teóricas en el estudio de la RSC, en esta sección se presentan brevemente las ideas centrales de éstas.

En la primera, Husted y Salazar (2006) muestran formalmente que un ejercicio estratégico de RSC significa un desplazamiento hacia abajo en la función de costos y uno hacia arriba en la función de beneficios esperados, y que esto no sólo implicaría un mejor resultado de dicho gasto sobre la utilidad de la empresa, sino también un mayor producto social. En la figura I se muestra este resultado.

Donde $\mathrm{BME}_{\mathrm{cs}}$ es el beneficio marginal estratégico de una unidad adicional de producto social, $\mathrm{CME}_{\mathrm{cs}}$ es el costo marginal estratégico de una unidad adicional de producto social y producto social es el resultado en términos de mejora social producido por el gasto en RSC.

La figura I muestra que en el caso estratégico existen beneficios adicionales que las empresas extraen de un nivel dado de producto social y que no son alcanzados por la empresa bajo coerción o en el caso altruista. Estudios que analizan los efectos en las relaciones de la empresa con los grupos relacionados de 
Figura I

INVERSIÓN SOCIAL ÓPTIMA EN EL CASO DE RESPONSABILIDAD SOCIAL ESTRATÉGICA

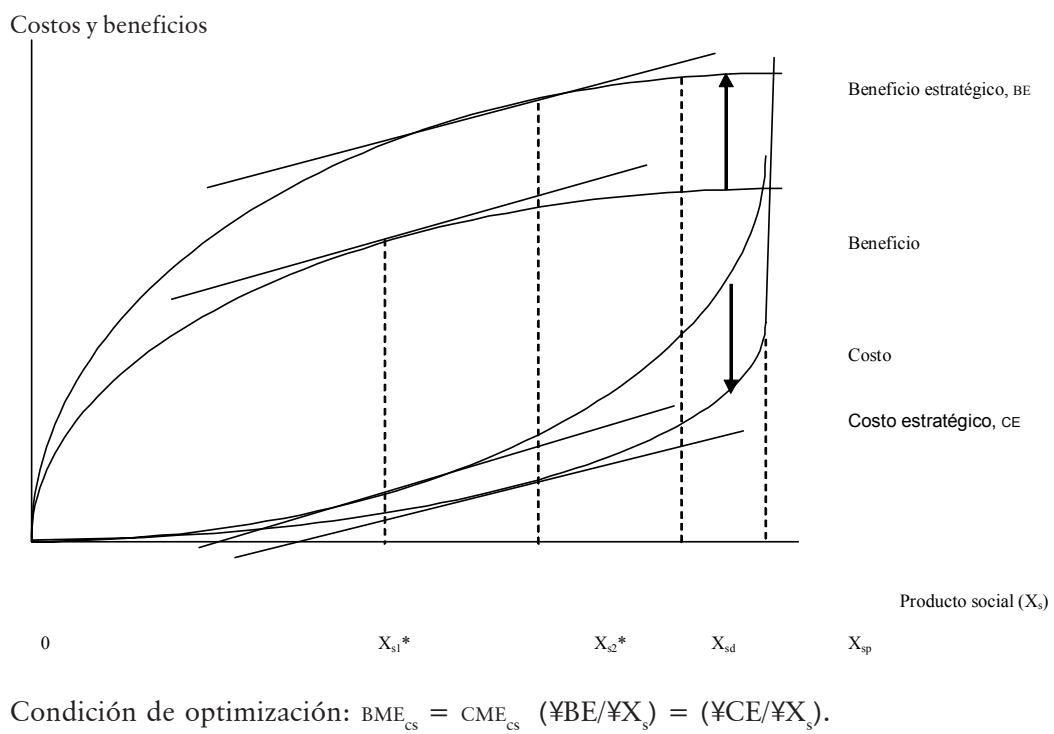

interés (stakeholders) sugieren que un enfoque estratégico hacia estos grupos puede traer efectos positivos en el desempeño financiero (Berman et al., 1999; Ogden y Watson, 1999). Se estima que a medida que aumenta el producto social, los beneficios marginales del mismo se reducirán, tanto si hablamos de la ventaja competitiva que esta inversión da a la empresa, como el beneficio que produce a la sociedad, esto debido a que, generalmente, desde que se alcanzan las últimas unidades de producto social (por ejemplo, llegar a las últimas personas por alfabetizar y a los últimos elementos contaminantes por evitar) hasta que se obtiene el producto social potencial $\left(\mathrm{X}_{\mathrm{sp}}\right)$, implicaría esfuerzos marginales mayores y beneficios adicionales muy pequeños para la empresa. Por lo que dado el comportamiento esperado en las funciones, existiría un nivel de producto social óptimo, que si bien no llega a alcanzar al producto social potencial, sí permite un nivel de producto que llevaría al mayor monto de rentabilidad en la inversión y un nivel positivo de producto social, mismo que puede o no coincidir con el producto social deseable $\left(\mathrm{X}_{\mathrm{sd}}\right)$ y que se espera difiera de país a país o de grupo a grupo dentro de una 
misma nación y que no podría fijarse en abstracto en un nivel determinado. ${ }^{4}$

La curva de costos se puede desplazar ante innovaciones que reduzcan el costo de producir el bien social. Se espera que esta función sea prácticamente vertical al nivel de $\mathrm{X}_{\mathrm{sp}}$, dado que ahí se alcanza el máximo de producto social, que es el mismo independientemente del tipo de motivación para el ejercicio de la responsabilidad social. "La cantidad óptima de producto social se alcanza cuando las pendientes de las curvas de costos y beneficios estratégicos son iguales. Dados los desplazamientos hacia arriba de la curva de beneficios y hacia debajo de la curva de costos, debidos al enfoque estratégico de la inversión social, la cantidad óptima de producto social aumentará" (Husted y Salazar, 2006: 84-85).

Siguiendo un enfoque marginalista, Husted y Salazar (2006) establecen un modelo básico (ver anexo) sobre los efectos de la inversión en RSC en la utilidad de la empresa y la mejora social, y demuestran formalmente que bajo el comportamiento esperado (figura I) el producto social bajo el enfoque estratégico en efecto resulta mayor que bajo motivaciones diferentes, llámense altruistas o bajo coerción, lo cual se espera correspondan más a comportamientos que no buscan necesariamente la utilidad privada, sino la social en el caso altruista, y el menor nivel de gasto en RSC para evitar multas o boicots, en el caso coercitivo.

Estos resultados son importantes y sugerentes para la empresa en términos de ejercer su responsabilidad social, lo que también implica reducir los problemas sociales que frecuentemente acompañan al sistema de mercado. El caso estratégico provee así una justificación para que las empresas privadas sean proactivas y contemplen en su presupuesto un plan de acción social, aun antes de iniciar un nuevo negocio.

También es interesante comparar el caso estratégico con el altruista. En este último, pueden o no ocurrir cambios en las curvas de costo y beneficio, pero es menos probable dado que la intención primaria no es obtener utilidad. A diferencia de la em-

\footnotetext{
${ }^{4}$ Con respecto a la medición del producto social existen diferentes formas de aproximación. En la teoría económica mucho se ha avanzado en este aspecto en el campo de la evaluación social de proyectos y valoración ambiental, donde se utilizan métodos como el de tasas sociales de descuento, valuación contingente, costos de viaje, precios hedónicos y costos evitados, entre otros. Para una mayor referencia sobre estos métodos, véase Fontaine (1997) y Pearce y Turner (1990). Recientemente se están practicando enfoques más integrales sobre evaluación en lo que se ha denominado evaluación de impacto social, para mayor referencia véase Becker y Vanclay (2003).
} 
presa estratégica, la altruista no busca maximizar la utilidad por peso gastado, sino más bien llevar el producto social a un nivel cercano o igual a $\mathrm{X}_{\mathrm{sd}}$. Aun así, pueden existir circunstancias en las que el nivel de producto socialmente deseable -el cual la empresa altruista busca alcanzar- coincida con el nivel de maximización del producto social.

\subsection{Sobre la relación entre $\mathrm{RSC}$, utilidad y competencia}

Salazar y Husted (2005) utilizan herramientas de la teoría de juegos para modelar las relaciones esperadas entre el gasto en RSC y las utilidades esperadas por la empresa en diferentes escenarios de competencia. Los autores muestran que tanto el producto social como la utilidad esperada -como efecto del gasto en RSC- varían de un modelo de competencia a otro o dependiendo de las prácticas de imitación que se dan es este tipo de inversiones. También encuentran que si bien la decisión de participar en RSC puede ser difícil para empresas pioneras, para las subsiguientes en el mismo mercado en general la decisión sería participar, generando con ello mayores probabilidades de rendimientos negativos en la inversión en RSC para todos los participantes y no positivos para algunos. Este último resultado se modifica cuando los mercados son suficientemente grandes, en ellos las decisiones en equilibrio generalmente llevarán a que sólo unas empresas participen propiciando naturalmente un mercado de RSC que es sustentable.

\section{Metodología}

\subsection{Hipótesis, muestra y datos}

El instrumento de estudio fue desarrollado sobre la base de los principales elementos de la estrategia social de la empresa. Las hipótesis se desprenden sobre todo de los planteamientos teóricos de Husted y Salazar (2005, 2006), estas hipótesis son: 1) la participación en acciones de RSC le significa a la empresa ganancias privadas; 2) diferentes formas de practicar la RSC le da a la empresa resultados privados distintos, la de corte estratégico se espera esté relacionada positivamente con la utilidad, la coercitiva en forma negativa y la altruista que no muestre una relación estadísticamente importante; 3) diferentes formas de practicar la RSC significarán para la empresa distintos resultados sociales y 
4) diferentes niveles de competencia entre las empresas llevan a resultados privados distintos según el sesgo en el ejercicio de la RSC. Se integraron preguntas orientadas a medir la estructura de Lee Burke y Jeanne Logsdon (1996) sobre la estrategia social de la empresa y de éstas se extrajeron y agruparon elementos que pueden llevar al diseño de índices de responsabilidad social. Se consideró información financiera obtenida de los datos que publica la revista Expansión sobre las 500 empresas mexicanas más importantes. La base de datos es la misma que se utilizó en el estudio de Husted y Salazar (2005).

La validez del instrumento se determinó por un análisis detallado de 10 personas del ámbito académico y de los negocios en México y España, quienes revisaron las diferentes partes del mismo. El cuestionario se envió en 1999 por correo al director general de cada una de las empresas de "Las 500 de Expansión" (Husted y Salazar, 2005: 12-13).

El método de aplicación de encuestas por correo cuenta con ventajas y desventajas, como señalan Delbert Miller y Neil Salkind (2002: 301), las principales desventajas son: el bajo porcentaje de respuesta, el esfuerzo requerido para aumentar la muestra después de la primer corrida y el problema de autoselección, el cual puede llevar a que el grupo que contesta tenga un perfil diferente al de la población. En contraparte, los mismos autores atribuyen a este método las ventajas de un menor costo, una mayor cobertura geográfica, llega a personas que regularmente son de difícil contacto -en este caso los directores generales de las empresas grandes-, permite respuestas más completas que las que se alcanzan bajo otros métodos, es más efectivo en situaciones que requieren consultar información con diversas personas, da a quienes responden un sentido de privacidad y disminuye el efecto de sesgo que el entrevistador puede inducir.

Tal como se esperaba en términos de lo señalado por Miller y Salkind (2002), la respuesta fue baja: en una primer corrida se recibió respuesta sólo de 40 empresas; luego, con seguimiento telefónico, se consiguió respuesta de otras 12 y con ello la muestra final incluyó 52 empresas.

\footnotetext{
${ }^{5}$ Anualmente la revista Expansión presenta un listado de las 500 empresas más grandes de México; dentro de este grupo aparecen empresas tanto del sector industrial como de servicios en sus diferentes ramas. En el subgrupo de las empresas que contestaron el cuestionario aplicado durante los trabajos del presente estudio, el promedio de empleados fue de 7,038 y formaban parte de los siguientes sectores: comercio, celulosa, petroquímica, construcción, petróleo, acero, cemento, minería, química, alimentos, papel, automotriz, aluminio y fertilizantes, entre otros.
} 
Debido a la baja tasa de respuesta, existe la posibilidad de sesgo por no respuesta. Una comparación entre quienes contestaron rápido con los que lo hicieron más tarde demuestra que no hay diferencia significativa en términos del tamaño de la empresa, en sus resultados financieros ni en sus respuestas al cuestionario. Algunos analistas sugieren que quienes responden tarde son muy parecidos a los que no responden (Armstrong y Overton, 1977). El hecho de que no existan diferencias importantes en las respuestas de quienes contestaron pronto y tarde sugiere que el problema de sesgo por no respuesta no es un problema significativo en este caso (Husted y Salazar, 2005: 12).

Reconocemos que 52 empresas son pocas para hacer generalizaciones, por ello, este trabajo contempla un análisis exploratorio de las relaciones teóricas planteadas en la bibliografía reciente. Como señalan Jorge Padua et al., "los estudios exploratorios son preponderantes en áreas o disciplinas en donde las problemáticas no están suficientemente desarrolladas" (2001: 31). En el mismo tenor, Roberto Hernández et al. indican: "Los estudios exploratorios se efectúan, normalmente, cuando el objetivo es examinar un tema o problema de investigación poco estudiado, del cual se tienen muchas dudas o no se ha abordado antes" (2003: 115). Como se planteó en la introducción, tal es el caso de la responsabilidad social corporativa y en particular en el ejercicio de ésta en Latinoamérica y México. Padua et al. agregan: "las muestras no probabilísticas resultan de importancia en las etapas exploratorias de la investigación” (2001: 83).

$\mathrm{Al}$ calcular el tamaño de la muestra se observa que, de acuerdo con el INEGI (1999), la cantidad de empresas grandes en México fue de $6,775.6$ Con esta población $\mathrm{N}$ y empleando un intervalo de confianza de $95.5 \%$ equivalente a 2 sigma; proporciones $\mathrm{p}$ y q de 0.88 y 0.12 , que de acuerdo con Lara (2000) representan las proporciones de empresas que en México invierten en causas sociales y las que no, respectivamente, y considerando un error estándar (E) de 9\%; al ensayar un muestreo simple para universos finitos menores a 100,000 observaciones, aplicamos la siguiente fórmula: ${ }^{7}$

$$
(4 \times \mathrm{N} \times \mathrm{p} \times \mathrm{q}) /\left[\mathrm{E}^{2}(\mathrm{~N}-1)+4 \times \mathrm{p} \times \mathrm{q}\right]=\mathrm{n}
$$

\footnotetext{
${ }^{6}$ Se consideran empresas manufactureras grandes aquéllas con más de 500 empleados y las de comercio y servicios con más de 100 empleados. Diario Oficial de la Federación, 30 de marzo de 1999, pp. 5-6.

7 Véase Lozano (1998).
} 
se obtiene una muestra $\mathrm{n}$ igual a 52 empresas:

$$
(4 \times 6775 \times 88 \times 12) /\left[9^{2}(6774)+(4 \times 88 \times 12)\right]=51.76 .
$$

$\mathrm{Al}$ verificar las respuestas se encontró que las proporciones de $\mathrm{p}$ y q eran de 0.865 y 0.135 , muy similares a las esperadas. Este resultado contribuye a eliminar el problema de autoselección del que se podría sospechar al aplicar cuestionarios por correo y donde los elementos de la muestra no fueron elegidos de manera aleatoria. Cabe mencionar que el error estándar que se contempla de $9 \%{ }^{8}$ puede parecer grande ante el típico error de $5 \%$ muy frecuentemente empleado en estudios sociales, sin embargo, aún 10\% suele ser aceptable. También es importante considerar lo que indica Sabrina Salazar cuando se refiere a los estudios empíricos sobre responsabilidad social corporativa: "aunque el tamaño reducido de la muestra puede representar una limitación para la generalización de resultados, es importante considerar que este problema es común en los estudios del área" (2004: 33). El propio estudio de esta autora observó una muestra de 18 empresas, lo que refleja el incipiente avance en la investigación formal de esta área de conocimiento. Un ejemplo más de lo difícil que es conseguir muestras mayores en esta área de estudio lo da la encuesta realizada por Briseida Lavielle (1998) del grupo Expansión, acerca de la filantropía empresarial, en el que de un total de 4,000 empresas a las que se les enviaron cuestionarios, solamente 64 respondieron.

\subsection{Las variables y su medición}

La motivación en la RSC se aproxima a través de la construcción de índices. Esta propuesta consiste en crear un índice para cada uno de los tipos de orientación que puede adoptar el ejercicio de responsabilidad social de la empresa: estratégico, coercitivo y altruista.

En la construcción de estas variables se contempló el uso de índices sumatorios simples, mismos que Padua et al. (2001: 41) recomiendan emplear cuando: a) las mediciones son resultado de una combinación de puntajes adjudicados arbitrariamente, $b$ ) los puntajes arbitrarios son indicadores de algunas dimensiones que queremos expresar en una cifra única y c) cuando el concep-

\footnotetext{
${ }^{8}$ Esto implica que en 91 de cada 100 veces los estimados encontrados estarán en un intervalo de confianza que comprenda el valor del parámetro poblacional. En ciencias sociales lo más usual es un error estándar de 5\%.
} 
to no es escalable, es decir, cuando no podemos asumir la existencia de un continuo subyacente, características presentes en las variables contempladas en el instrumento.

En la tabla 1 se incluyen los conceptos y se calcula el rango de valores referentes al índice para el caso de la contribución altruista.

Como se puede observar en la tabla 1 , el índice toma valores entre 5 y 40 . Donde 40 representa una orientación altamente altruista y 5 una no altruista.

Siguiendo el procedimiento anterior, en la tabla 2 se incluyen los conceptos y se calcula el rango de valores referentes al índice para el caso de la contribución social bajo coerción.

El índice de la contribución social del tipo coercitivo toma valores entre 5 y 25 , donde 25 representa una orientación altamente coercitiva y 5 una no coercitiva.

En la tabla 3 se incluyen los conceptos y se calcula el intervalo de valores referentes al índice para el caso de la contribución social estratégica.

El índice de la contribución social de tipo estratégica toma valores entre 25 y 115 . Donde 115 representa una orientación altamente estratégica y 25 una no estratégica.

En la tabla 4 se incluyen los conceptos y se calcula el rango de valores referentes al índice del nivel de competencia que enfrenta la empresa.

El índice de competencia toma valores entre 12 y 65 . Donde 65 representa un nivel alto de competencia que enfrenta la empresa y 12 uno muy bajo o nulo en los mercados principales donde la empresa participa.

Este índice contempla cinco componentes que coinciden con el de responsabilidad social estratégica, por ello, a la hora de relacionarlo en las pruebas estadísticas, se usará un índice de competencia ajustado, mismo que no contemplará estos componentes y cuyo rango de valores va de 2 a 45 .

\subsection{Técnicas de análisis}

Las hipótesis estarán sujetas a prueba empleando coeficientes de correlación simple y la técnica de mínimos cuadrados ordinarios del análisis de regresión, con los indicadores financieros de retorno sobre activos y retorno sobre capital como variables dependientes. Entre las variables independientes se proponen cuatro índices, uno por cada tipo de ejercicio de la responsabilidad social de la empresa y uno más para el nivel de competencia que 
Tabla 1

Variables incluidas en el cálculo del índice de responsabilidad social altruista

\begin{tabular}{|c|c|c|c|}
\hline $\begin{array}{l}\text { Pregunta incluida } \\
\text { en el cuestionario } \\
\text { a empresas }\end{array}$ & Inciso & $\begin{array}{l}\text { Valores } \\
\text { posibles }\end{array}$ & $\begin{array}{l}\text { Totales } \\
\text { posibles }\end{array}$ \\
\hline \multirow{4}{*}{$\begin{array}{l}\text { 4. De las siguientes posibles } \\
\text { motivaciones de participa- } \\
\text { ción en programas de } \\
\text { interés social, por favor } \\
\text { indique qué tanto coinciden } \\
\text { con las de su empresa. }\end{array}$} & $\begin{array}{l}\text { 1. Hay que adaptarse a } \\
\text { los valores emergentes } \\
\text { de la sociedad. }\end{array}$ & $1-5$ & \\
\hline & $\begin{array}{l}\text { 2. La empresa debe } \\
\text { regresar parte de lo que } \\
\text { gana a la sociedad. }\end{array}$ & $1-5$ & \\
\hline & $\begin{array}{l}\text { 7. Por compromisos } \\
\text { personales de los altos } \\
\text { directivos. }\end{array}$ & $1-5$ & \\
\hline & $\begin{array}{l}\text { 17. Por responsabilidad } \\
\text { social. }\end{array}$ & $1-5$ & $4-20$ \\
\hline $\begin{array}{l}\text { 6. ¿Se lleva a cabo alguna } \\
\text { medición de los resultados } \\
\text { del otorgamiento de estas } \\
\text { ayudas sociales? }\end{array}$ & Sí o no. & 0 о 5 & 0 о 5 \\
\hline $\begin{array}{l}\text { 7. ¿Cómo se modifica el } \\
\text { plan de ayudas sociales de } \\
\text { un año dado en términos } \\
\text { de los resultados económico- } \\
\text { financieros de la empresa } \\
\text { durante el año? }\end{array}$ & $\begin{array}{l}\text { 4. Las ayudas se deter- } \\
\text { minan con base en las } \\
\text { necesidades de los } \\
\text { beneficiarios y no de la } \\
\text { marcha del negocio. }\end{array}$ & 0 о 5 & 0 o 5 \\
\hline $\begin{array}{l}\text { 12. ¿ La empresa realiza } \\
\text { estudios sociales que le } \\
\text { permitan detectar } \\
\text { directamente las áreas de } \\
\text { oportunidad de sus } \\
\text { programas de ayuda social? }\end{array}$ & Sí o no. & 0 о 5 & 0 о 5 \\
\hline $\begin{array}{l}\text { 13. ¿Son importantes los } \\
\text { siguientes objetivos en la } \\
\text { misión de su empresa? }\end{array}$ & $\begin{array}{l}\text { 10. Ayudar a causas } \\
\text { sociales. }\end{array}$ & $1-5$ & $1-5$ \\
\hline Totales (rango) & & & $5-40$ \\
\hline
\end{tabular}

Fuente: Elaboración propia. 
Tabla 2

Variables incluidas en el cálculo del índice de responsabilidad social coercitiva

\begin{tabular}{|c|c|c|c|}
\hline $\begin{array}{l}\text { Pregunta incluida } \\
\text { en el cuestionario } \\
\text { a empresas }\end{array}$ & Inciso & $\begin{array}{c}\text { Valores } \\
\text { posibles }\end{array}$ & $\begin{array}{l}\text { Totales } \\
\text { posibles }\end{array}$ \\
\hline \multirow{4}{*}{$\begin{array}{l}\text { 4. De las siguientes posibles } \\
\text { motivaciones de participa- } \\
\text { ción en programas de } \\
\text { interés social, por favor } \\
\text { indique qué tanto coinciden } \\
\text { con las de su empresa. }\end{array}$} & $\begin{array}{l}\text { 6. Para mejorar la imagen } \\
\text { de la empresa ante la } \\
\text { opinión pública. }\end{array}$ & $1-5$ & \\
\hline & $\begin{array}{l}\text { 10. Para cumplir con } \\
\text { requisitos legales. }\end{array}$ & $1-5$ & \\
\hline & $\begin{array}{l}\text { 12. Para facilitar la } \\
\text { implantación de la empresa } \\
\text { en la comunidad. }\end{array}$ & $1-5$ & \\
\hline & $\begin{array}{l}\text { 13. Para mejorar las } \\
\text { relaciones con entidades } \\
\text { públicas. }\end{array}$ & $1-5$ & $4-20$ \\
\hline $\begin{array}{l}\text { 9. Los programas de interés } \\
\text { social de la empresa son } \\
\text { conocidos: }\end{array}$ & 3. Por el gobierno. & $1-5$ & $1-5$ \\
\hline Totales (rango) & & & $5-25$ \\
\hline
\end{tabular}

Fuente: Elaboración propia. 
Tabla 3

\section{Variables incluidas en el cálculo del índice de responsabilidad social estratégica}

\begin{tabular}{|c|c|c|c|}
\hline $\begin{array}{l}\text { Pregunta incluida } \\
\text { en el cuestionario }\end{array}$ & Inciso & $\begin{array}{l}\text { Valores } \\
\text { posibles }\end{array}$ & $\begin{array}{l}\text { Totales } \\
\text { posibles }\end{array}$ \\
\hline a empresas & & & \\
\hline \multirow{13}{*}{$\begin{array}{l}\text { 4. De las siguientes posibles } \\
\text { motivaciones de participa- } \\
\text { ción en programas de } \\
\text { interés social, por favor } \\
\text { indique qué tanto coinciden } \\
\text { con las de su empresa. }\end{array}$} & $\begin{array}{l}\text { 3. Para influir en las } \\
\text { decisiones de compra } \\
\text { de mis clientes. }\end{array}$ & $1-5$ & \\
\hline & $\begin{array}{l}\text { 4. Para desarrollar nuevos } \\
\text { negocios con objetivos } \\
\text { sociales. }\end{array}$ & $1-5$ & \\
\hline & $\begin{array}{l}\text { 5. Para que los trabajadores } \\
\text { estén orgullosos de la } \\
\text { empresa. }\end{array}$ & $1-5$ & \\
\hline & $\begin{array}{l}\text { 8. Es publicitariamente } \\
\text { rentable. }\end{array}$ & $1-5$ & \\
\hline & $\begin{array}{l}\text { 9. Para captar nuevos } \\
\text { clientes. }\end{array}$ & $1-5$ & \\
\hline & $\begin{array}{l}\text { 11. Para mantener clientes } \\
\text { actuales. }\end{array}$ & $1-5$ & \\
\hline & $\begin{array}{l}\text { 14. Es una forma de } \\
\text { diferenciar la empresa de } \\
\text { los competidores. }\end{array}$ & $1-5$ & \\
\hline & $\begin{array}{l}\text { 15. Es rentable incluso en } \\
\text { el corto plazo. }\end{array}$ & $1-5$ & \\
\hline & $\begin{array}{l}\text { 16. Para desarrollar nuevos } \\
\text { productos. }\end{array}$ & $1-5$ & \\
\hline & $\begin{array}{l}\text { 19. Es necesario el } \\
\text { bienestar social general } \\
\text { para que la empresa } \\
\text { sobreviva en el largo plazo. }\end{array}$ & $1-5$ & \\
\hline & $\begin{array}{l}\text { 20. Para abrir nuevos } \\
\text { mercados. }\end{array}$ & $1-5$ & \\
\hline & $\begin{array}{l}\text { 21. Contar con una fuerza } \\
\text { de trabajo más capacitada. }\end{array}$ & $1-5$ & \\
\hline & $\begin{array}{l}\text { 22. Para obtener un } \\
\text { tratamiento fiscal favorable. }\end{array}$ & $1-5$ & $13-65$ \\
\hline $\begin{array}{l}\text { 6. ¿Se lleva a cabo alguna } \\
\text { medición de los resultados } \\
\text { del otorgamiento de estas } \\
\text { ayudas sociales? }\end{array}$ & No o sí. & 0 о 5 & 0 o 5 \\
\hline $\begin{array}{l}\text { 7. ¿Cómo se modifica el } \\
\text { plan de ayudas sociales de } \\
\text { un año dado en términos } \\
\text { de los resultados econó- }\end{array}$ & $\begin{array}{l}\text { 1. Se respetan los } \\
\text { presupuestos originales } \\
\text { sin importar la marcha } \\
\text { del negocio. }\end{array}$ & 0 о 5 & 0 о 5 \\
\hline $\begin{array}{l}\text { mico-financieros de la } \\
\text { empresa durante el año? }\end{array}$ & $\begin{array}{l}\text { 2. Si durante el año la } \\
\text { rentabilidad del negocio }\end{array}$ & 0 о 5 & 0 о 5 \\
\hline
\end{tabular}


continuación...

\begin{tabular}{|c|c|c|c|}
\hline $\begin{array}{c}\text { Pregunta incluida } \\
\text { en el cuestionario } \\
\text { a empresas }\end{array}$ & Inciso & $\begin{array}{c}\text { Valores } \\
\text { posibles }\end{array}$ & $\begin{array}{c}\text { Totales } \\
\text { posibles }\end{array}$ \\
\hline
\end{tabular}

va rezagada $v s$. lo esperado, las ayudas se reducen.

9. Los programas de interés 1. Por la comunidad social de la empresa son objetivo de la ayuda.

$1-5$ conocidos:

10. Los programas de la empresa se difunden:

12. ¿La empresa realiza estudios sociales que le permitan detectar directamente las áreas de oportunidad de sus programas de ayuda social? 14. Considera que el cumplimiento de los objetivos sociales de la empresa están:

Totales (rango)

Fuente: Elaboración propia.
1. Ligados al cumplimiento de los objetivos de rentabilidad.
$25-115$
2. Por los trabajadores de la empresa.

4. Por otras instituciones que prestan ayudas similares.

1. Dentro de la misma empresa.

2. Mediante una página

de Internet.

4. Directamente en la comunidad objetivo.

Sí o no.

1-5 3-15

5 о $0 \quad 5$ о 0

$1-5 \quad 1-5$ 
Tabla 4

\section{Variables incluidas en el cálculo del índice de competencia}

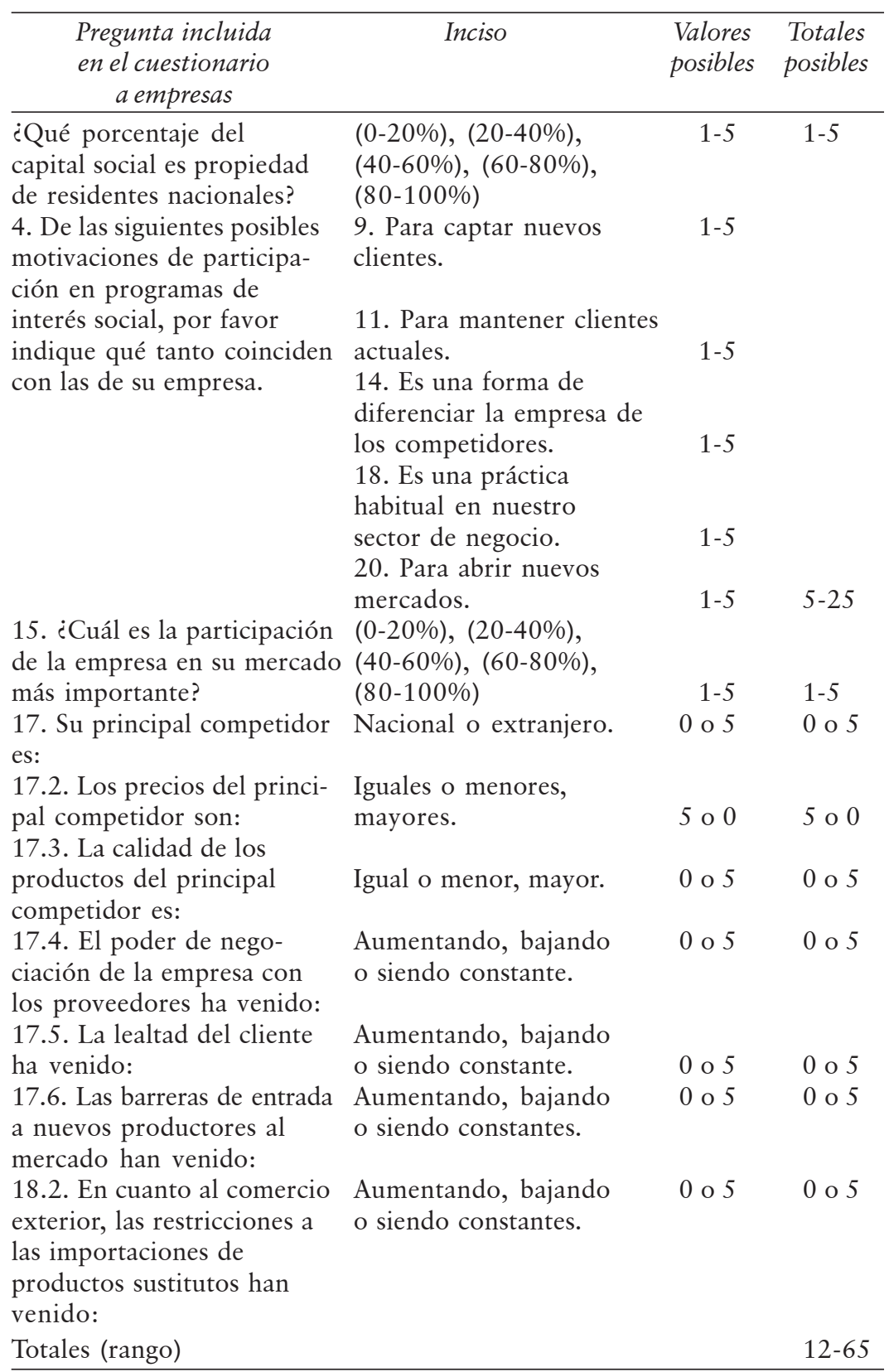

Fuente: Elaboración propia. 
Economía, Sociedad y Territorio, vol. vIII, núm. 27, 2008, 739-768

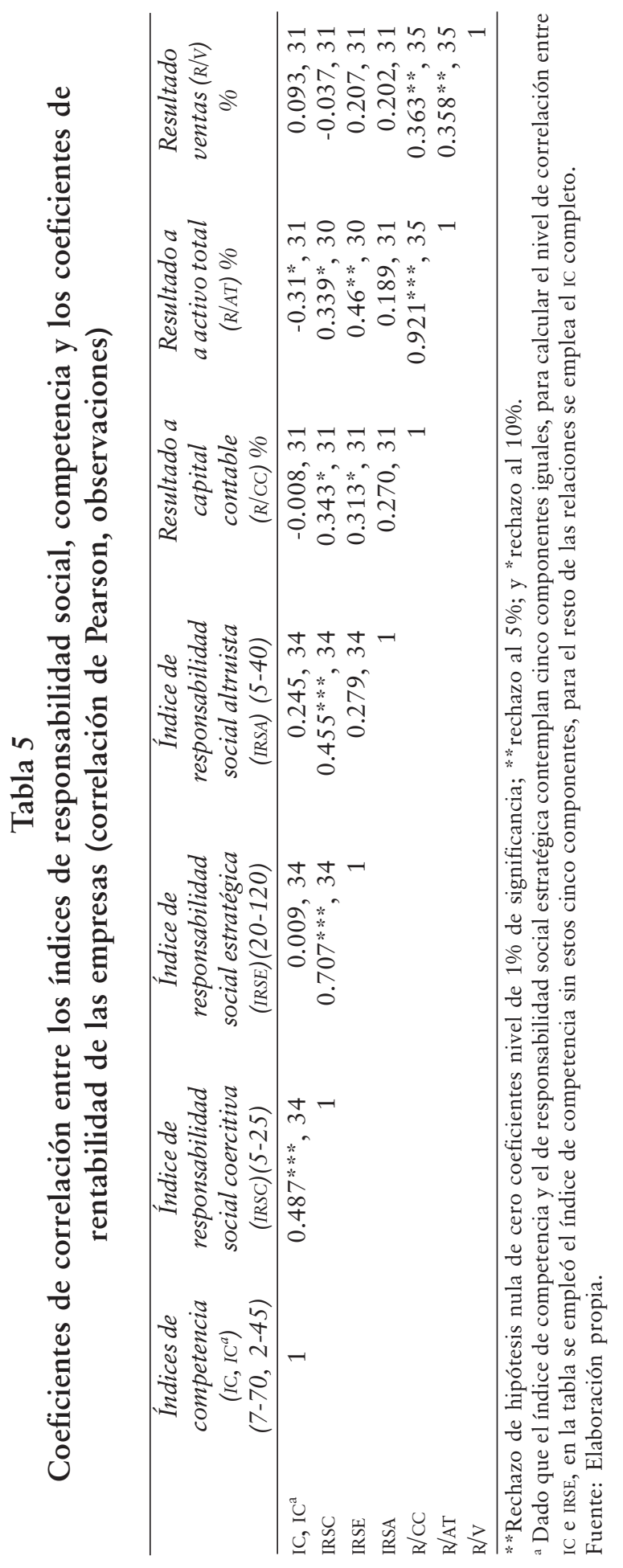


la misma enfrenta, estos índices también se relacionarán estadísticamente con los indicadores de rentabilidad privada.

\section{Resultados}

Se realizó un análisis de correlación entre los índices de responsabilidad social, el índice de competencia y los coeficientes de rentabilidad, los resultados aparecen en la tabla 5 .

En cuanto a las relaciones esperadas, se puede observar que el índice de responsabilidad social estratégica tiene una relación estadística significativa con la rentabilidad, esta última medida como el coeficiente de resultado a capital contable o bien como resultado a activo total, asimismo, el índice de responsabilidad social altruista no mostró relación con los coeficientes de rentabilidad. Estos resultados están de acuerdo con lo esperado teóricamente.

También se encuentra una relación estadística significante entre la competencia y el índice de responsabilidad social coercitiva, la cual fue como se esperaba en teoría y de acuerdo con lo que propone David Baron (2001: 31). Por su parte, siguiendo el sentido teórico, el índice de competencia no observa una relación estadística significante con el de responsabilidad social altruista, pero en su versión ajustada sí se relaciona de manera inversa con el de rentabilidad $(\mathrm{r}=-0.31, \mathrm{p}=0.09, \mathrm{n}=31)$, este último resultado coincide con el que encontraron Chávez et al. (2000: 46) cuando consideraron 28 complejos industriales mexicanos, con base en la información del censo industrial de 1993.

De acuerdo con Baron (2001: 31), también se esperaría que aquellas empresas que sienten más la posibilidad de coerción social en su contra y que al mismo tiempo enfrentan alta competencia, podrían participar más fácilmente en inversiones sociales, más allá de las que surgirían de la lógica pura del mercado. En este sentido se encontraron relaciones estadísticamente significativas y de signo esperado, entre la variable que describe en qué medida el ejercicio de la responsabilidad social de la empresa es una práctica habitual dentro de la industria donde se encuentra y el índice de contribución social bajo coerción $(\mathrm{r}=-0.38, \mathrm{p}=$ $0.04, \mathrm{n}=31$ ), así como con el índice del nivel de competencia que enfrenta la empresa $(\mathrm{r}=-0.37, \mathrm{p}=0.04, \mathrm{n}=31)$.

Se probaron las relaciones teóricas con base en regresiones lineales empleando el método de mínimos cuadrados ordinarios, al incluir entre las variables explicativas los diferentes índices de 
responsabilidad social, inicialmente no se encontraron ecuaciones libres de problemas estadísticos. En éstas primeras ecuaciones se presentó el problema de multicolinealidad que ya se esperaba dada la alta relación entre los índices de responsabilidad social coercitiva con el de competencia y con el de responsabilidad social estratégica, dado que en estas estimaciones iniciales el índice de responsabilidad coercitiva era menos importante, se eliminó, dejando las variables correlacionadas con éste para estimar funciones subsiguientes, así se llegó a ecuaciones más significativas:

$$
\mathrm{R} / \mathrm{V}=3.614+0.213 \mathrm{IRSE}-0.350 \mathrm{IC}^{\mathrm{a}}+\mathrm{H}
$$

Valores de p: (0.0510) (0.0081) (0.0630)

$\mathrm{R}^{2}$ ajustada $=0.257 ; \mathrm{DW}=2.65$

$$
\mathrm{R} / \mathrm{V}=3.213+0.258 \text { IRSE }-0.409 \mathrm{IC}^{\mathrm{a}}-0.0093 \mathrm{PO}+\mathrm{H}
$$

Valores de p: (0.0492) (0.002) (0.026) (0.068)

$\mathrm{R}^{2}$ ajustada $=0.370 ; \mathrm{DW}=2.27$

En la primera ecuación, tanto el índice de competencia ajustado (IC ${ }^{a}$ como el de responsabilidad social estratégica (IRSE) explican la variable de rentabilidad, esta última expresada por la razón de resultado a ventas (R/v). Al colocar como variable dependiente el IRSE e incluir $\mathrm{R} / \mathrm{V}$ como variable explicativa, el modelo no es estadísticamente significativo. Esto podría sugerir que la rentabilidad se determina por la orientación estratégica del ejercicio de la responsabilidad social, pero no al revés. ${ }^{9}$ Aún así, el resultado referente a la causalidad se debe analizar con más detalle con información para más de un momento.

En la segunda ecuación se controló por personal ocupado (PO), variable que suele constituir uno de los criterios para distinguir el tamaño de la empresa. En este caso, el modelo es estadísticamente mejor que el anterior, aunque el signo esperado en el coeficiente de relación de la nueva variable con la rentabilidad podría no ser el esperado, es decir, a mayor tamaño se pensaría que la empresa tiene mejor posición competitiva y esto podría permitirle más rentabilidad, pero esto no es así en el modelo estimado. Nuevamente vemos que al controlar por competencia y personal ocupado, la variable de IRSE sigue siendo significativa para explicar los cambios en el resultado sobre ventas.

Algo que permanece casi inexplorado tanto en México como en cualquier otro país, es el efecto que la acción social de las

${ }^{9}$ Esta dirección de la causalidad se aborda poco en el trabajo empírico de esta área del conocimiento. 
empresas tiene sobre los beneficiarios y la relación entre este efecto y la rentabilidad de la propia empresa. De acuerdo con lo observado, en este grupo de empresas residentes en México, 36\% informaron que hacen algún tipo de medición de los efectos sociales de sus programas y $21 \%$ dijo tener mecanismos para detectar nuevas necesidades sociales.

Medir los efectos sociales no es tarea fácil y las herramientas y métodos de medición en este campo, si bien han progresado, aún enfrentan retos importantes, como lo considera Frank Vanclay (2003: 8) cuando habla en particular de la evaluación de impacto social, sobre la cual menciona que todavía cuenta con muy pocas técnicas de estudio. Si bien es cierto que hay una gran experiencia en la medición de resultados sociales producto de proyectos gubernamentales, esta experiencia no se aplica de la misma forma cuando los programas provienen de inversiones privadas y cuando también se busca incluir relaciones sociales. Para aproximar los efectos sociales, en este estudio se consideró como variable proxy de los mismos el cociente de inversión en programas sociales a valor de las ventas anuales de la empresa, aunque la variable dista de ser una representación clara de los beneficios totales que el programa social pudiera haber producido, se considera que estos beneficios guardan una relación directa con dicha inversión.

Se encontró una relación negativa y estadísticamente significante entre la variable de inversión social a ventas con el índice de coerción $(\mathrm{r}=-0.41, \mathrm{p}=0.03, \mathrm{n}=29)$ y con los cocientes de resultado a capital $(\mathrm{r}=-0.40, \mathrm{p}=0.04, \mathrm{n}=27)$ y resultado a activo total $(\mathrm{r}=-0.035, \mathrm{p}=0.07, \mathrm{n}=27)$. Lo cual resulta congruente con la idea de que quien ejerce su responsabilidad social bajo coerción tiende a minimizar su nivel de inversión social, del cual normalmente sólo derivan pérdidas netas.

\section{Conclusiones}

La tabla 6 muestra las hipótesis de trabajo y las conclusiones a que se puede llegar con base en las estimaciones realizadas.

Como observa María Lara (2000), todavía son pocas las empresas que muestran mayor sesgo hacia un estilo de contribución social de corte estratégico, aun cuando hay elementos que indican con claridad que los aportes no son principalmente altruistas, por su parte, no hay evidencia para establecer que cuen- 
Tabla 6

Hipótesis de trabajo y resultado de su contraste estadístico

Hipótesis
1. La participación en acciones
de RSc le significa a la empresa
ganancias privadas.
2. Diferentes formas de la
empresa en prácticas de RSC le
significan diferentes resultados
privados.

La sola participación de la empresa en acciones de RSC no se encontró estadísticamente asociada con la generación de mayores niveles de rentabilidad. Esto hace rechazar la hipótesis. El sesgo estratégico en los gastos implícitos en la participación de la empresa en acciones de RSC se relacionó positivamente con la generación de mayores niveles de rentabilidad, por su parte, el sesgo coercitivo se relacionó con menor rentabilidad y el altruista no mostró relación estadísticamente significante. Esto permite aceptar la hipótesis.

3. Diferentes formas de la Se encuentra una correlación negativa empresa en prácticas de RSC entre el producto social y dos coeficientes significarán diferentes resultados de rentabilidad, así como con el índice de sociales. sesgo coercitivo del gasto en RSC. Por su parte, el producto social no se encontró asociado estadísticamente con el sesgo estratégico. Esta evidencia no permite aceptar la hipótesis.

4. Diferentes niveles de Se encontró que mayores niveles de competencia entre las empresas llevan a resultados privados competencia están asociados con mayores niveles de sesgo coercitivo y menores distintos según el sesgo en el ejercicio de la RSC. niveles de sesgo altruista en la motivación de gasto en RSC.

Esto hace aceptar la hipótesis.

Fuente: Elaboración propia. 
tan con un plan de apoyo social que les lleve a generar ventajas competitivas.

Una muestra de 52 empresas no alcanza a ser lo suficientemente representativa y es posible que su ampliación arroje evidencia más confiable. Aunque hay elementos para descartar el problema de autoselección, la encuesta sufre de las usuales debilidades para autoinformar datos (Abrahamson, 1983). Trabajos futuros, con muestras más grandes y representativas a lo largo de diferentes sectores con distintos niveles de rivalidad, aportarían evidencia útil en el diseño de política empresarial y pública sobre el tratamiento de la RSC.

La construcción de índices de responsabilidad social y de competencia son una nueva propuesta que permiten mostrar algunos resultados en la línea de lo expuesto teóricamente por Husted y Salazar, 2005, 2006). El uso de estos índices puede servir en otros estudios teóricos y empíricos. Como también señala Baron (2001: 39), la determinación del sesgo altruista, estratégico o ante amenazas que caracteriza las acciones de responsabilidad social de la empresa es importante para luego relacionarlo con las variables de desempeño financiero o bien de bienestar social que estas acciones producen. La discusión sobre la validez de estos índices -que combinan algunas de las variables tradicionalmente empleadas en el cálculo de coeficientes de concentración industrial con otras variables más cualitativas y que permiten incluir percepciones de los gerentes y administradores sobre el grado de competencia en sus principales mercados, comúnmente consideradas en los análisis con teoría de juegos- es algo que se espera motive trabajos futuros que coadyuven a alcanzar estimaciones más fuertes. También podrían servir para aportar nuevos elementos de decisión en los actuales procesos de certificación de empresas en RSC, donde en consonancia con las nuevas orientaciones teóricas en este campo, sería indispensable distinguir entre empresas con RSC altruista, estratégica o coercitiva, ya que como se ha mostrado el sesgo puede estar influyendo directamente en el resultado privado y social de dichas prácticas, lo que añadiría transparencia ante la sociedad sobre los efectos esperados y el tipo de compromiso social empresarial que en realidad se está practicando.

La proporción de quienes consideran que participan en programas de responsabilidad social es amplio, pero entre ellos la orientación no parece ser estratégica, lo cual, como establecen Husted y Salazar (2006), no es tan eficiente ni tan sustentable 
para ellos mismos y para la sociedad, como podría ser dicho aporte si tuviera planteamientos mejores y más estratégicos. La estimación de indicadores más completos de producto social también es algo que queda por integrar en futuros trabajos, las relaciones negativas aquí encontradas entre el producto social con el sesgo coercitivo en el gasto en RSC y los niveles de rentabilidad, hacen patente que dichos gastos no son del todo sustentables y quizá se requiera de un mejor análisis de políticas públicas y privadas para orientarlos de forma que aumente su probabilidad de generar una contribución social sustentable.

\section{Anexo}

A continuación se presentan las ecuaciones de costos y beneficios empleadas por Husted y Salazar (2006) y la forma en que los autores muestran la ventaja en utilidad privada y producto social cuando la inversión en RSC cuenta con un sesgo estratégico, más que uno altruista o bajo coerción. Se considera que los rendimientos marginales de la inversión social, al igual que los correspondientes con cualquier otro tipo de inversión de la empresa son decrecientes. Otro tipo de retornos y la consideración de producción de corto plazo no se consideran dado que en principio se intenta establecer un caso básico.

Primero se definen las funciones que representan las curvas de costo social y beneficio social bajo coerción:

Función de costos: $\mathrm{C}_{\mathrm{cs}}=\mathrm{D}_{1} \mathrm{x}^{\mathrm{O}}$. Donde: $\mathrm{O}>1 ; \mathrm{D}_{1}>0 ; \mathrm{y}$

Función de beneficios: $\mathrm{B}_{\mathrm{cs}}=\mathrm{A}_{1} \mathrm{x}^{\mathrm{D}}$. Donde: $0<\mathrm{D}<1$; $\mathrm{A}_{1}>0$ $\mathrm{x}=$ unidades de producto social.

$A_{1}, D_{1}$ son los coeficientes del caso de coerción, mientras $D$ y $\mathrm{O}$ son exponentes que adoptan valores constantes. $\mathrm{A}_{1}$ es el coeficiente de la relación que se da entre el producto social y los beneficios sociales de la empresa, mientras $\mathrm{D}_{1}$ es el coeficiente de la relación entre el producto social y el costo de los proyectos sociales que lleva a cabo la empresa.

Como se desprende del análisis gráfico, la maximización de utilidades o bien la minimización del costo neto, ${ }^{10}$ sucede al nivel de producto social que iguala las pendientes de las funciones de

${ }^{10}$ Esto en el caso de que la curva de costos siempre esté por arriba de la de beneficios. 
costos y beneficios totales. En el caso de coerción, podemos obtener ese nivel de producto social igualando las primeras derivadas de ambas funciones, a continuación se muestra el procedimiento y el resultado:

$$
\begin{aligned}
& \mathrm{C}_{\mathrm{cs}}{ }^{\prime}=\mathrm{B}_{\mathrm{cs}}{ }^{\prime} \text {; } \\
& \mathrm{C}_{\mathrm{cs}}^{\mathrm{cs}}=\mathrm{D}_{1}^{\mathrm{cs}} \mathrm{Ox}^{\mathrm{QD}}{ }^{1} \text {; } \\
& \mathrm{B}_{\mathrm{cs}}^{\mathrm{cs}}=\mathrm{A}_{1} \mathrm{Dx}^{\mathrm{D}-1} \text {; } \\
& \mathrm{D}_{1} \mathrm{Ox}=\mathrm{A}_{1} \mathrm{Dx}^{\mathrm{D}-1} \text {; } \\
& 1=\left(D_{1} \text { OA } A, D\right) x^{\mathrm{Oa}} \text {; } \\
& \mathrm{x}^{*}=\left(\mathrm{A}_{1} \mathrm{D} / \mathrm{D}_{1} \mathrm{O} \text { TسmODD }\right) \text {; }
\end{aligned}
$$

Así, el nivel de producto social que maximiza las utilidades que la empresa deriva de su ejercicio de la responsabilidad social bajo coerción, es $x^{*}=\left(A_{1} D_{/} D_{1} O\right)^{1 /(Q D D)}$.

Ahora, en el caso de responsabilidad social estratégica, las funciones de referencia serían:

Función de costos: $\mathrm{C}_{\mathrm{cs}}=\mathrm{D}_{2} \mathrm{x}^{\mathrm{O}}$;

Función de beneficios: $\mathrm{B}_{\mathrm{cs}}=\mathrm{A}_{2} \mathrm{x}^{\mathrm{D}}$;

Donde: $1>1 ; \mathrm{D}_{1}>\mathrm{D}_{2}>0$;

y $0<\mathrm{D}<1 ; \mathrm{A}_{2}>\mathrm{A}_{1}>0$.

Así, puede obtenerse que: $x_{2}{ }^{*}=\left(A_{2} D / D_{2} O\right)^{1 /(~ Q D D)}$.

Si la relación entre los coeficientes constantes (A, D, DएD) se mantiene como se ha supuesto, se demuestra que $\mathrm{x}_{2}{ }^{*}>\mathrm{x}_{1}{ }^{*}$, es decir, que el producto social óptimo es mayor en el caso RSC estratégica que bajo coerción. La comparación con el caso altruista también la presentan estos autores, pero no en forma matemática sino sólo gráfica, debido a que parte de la idea de que el altruista no está interesado en su utilidad, sino en la social y no existiría un signo de relación causal esperado a priori entre este tipo RSC y la utilidad privada.

\section{Bibliografía}

Abrahamson, Mark (1983), Social research methods, Nueva Jersey, Estados Unidos, Englewood Califfs-Prentice Hall.

Armstrong, J. Scott y Terry Overton (1977), "Estimating no response bias in mail surveys", Journal of Marketing Research,18(3), American Marketing Association, Chicago, pp. 396-402. 
Baron, David (2001), "Private politics, corporate social responsibility, and integrated strategy", Journal of Economics \& Management Strategy, 10(1), MIT Press, Cambridge, Massachussets, Estados Unidos, pp. 7-45.

Becker, Henk y Frank Vanclay (eds.) (2003), The international handbook of social impact assessment: Conceptual and methodological advances, Northampton, Massachussets, Estados Unidos, Edward Elgar.

Berman, Shawn, Andrew Wicks, Suresh Kotha y Thomas Jones (1999), "Does stakeholder orientation matter? The relationship between stakeholder management models and firm financial performance", The Academy of Management Journal, 42(5), Academy of Management, Ada, Ohio, Estados Unidos, pp. 488-506.

Burke, Lee y Jeanne Logsdon (1996), “How corporate social responsibility pays off", Long Range Planning, 29(4), Pergamon Press, Londres, Inglaterra, pp. 495-502.

Cemefi (Centro Mexicano para la Filantropía) (2004), en <http:// www.cemefi.org.mx>, 30 de noviembre de 2004.

Chávez, María, María García, Heliana Montserrat, Josefina Robles, Eunice Taboada y Leticia Velásquez (2000), Estudios sectoriales de las manufacturas mexicanas, México, Universidad Autónoma Metropolitana, Azcapotzalco.

Correa, María, Sharon Flynn y Amit Alon (2004), Responsabilidad social corporativa en América Latina: una visión empresarial, Santiago de Chile, CEPAL.

Feddersen, Timothy y Thomas Guilligan (2001), "Saints and markets: Activists and the supply of credence goods", Journal of Economics \& Management Strategy, 10(1), MIT Press, Cambridge, Massachussets, Estados Unidos, pp. 149-171.

Fontaine, Ernesto (1997), Evaluación social de proyectos, Santiago de Chile, Universidad Católica de Chile.

Griffin, Jennifer y John Mahon (1997), “The corporate social performance and corporate financial performance deba- 
te: Twenty-five years of incomparable research", Business and Society, 36(1), Business Research Center, College of Business Administration, Roosevelt University, Chicago, pp. 5-31.

Hernández, Roberto, Carlos Fernández y Pilar Baptista (2003), Metodología de la investigación, México, McGraw Hill.

Husted-Corregan, Bryan y José de Jesús Salazar-Cantú (2005), "Un estudio exploratorio sobre la estrategia social de empresas grandes ubicadas en México", Contaduría y Administración, 215, Universidad Nacional Autónoma de México, México, pp. 9-23.

Husted-Corregan, Bryan y José de Jesús Salazar-Cantú (2006), "Taking Friedman Seriously: Maximizing Profits and Social Performance", Journal of Management Studies, 43(1), Blackwell Publishing, Durham, Reino Unido, pp. 75-91.

INEGI (Instituto Nacional de Estadística, Geografía e Informática) (1999), Censos Económicos, México, INEGI.

Lara, María (2000), Filantropía empresarial: convicción y estrategia, México, Pax.

Lavielle, Briseida (1998), "Filantropía empresarial”, Expansión, 7 de octubre, México, pp. 11-12.

Lozano, José (1998), Curso-taller de apoyo metodológico a la investigación, México, Instituto Tecnológico y de Estudios Superiores de Monterrey.

Margolis, Joshua y James Walsh (2001), People and profits: The search for a link between a company's social and financial performance, Nueva Jersey, Estados Unidos, Lawrence Erlbaum Associates Publishers.

McWilliams, Abagail y Donald Siegel (2000), “Corporate social responsibility and financial performance: Correlation or misspecification?", Strategic Management Journal, 21(5), John Wiley \& Sons, Hoboken, Nueva Jersey, Estados Unidos, pp. 603-609. 
Miller, Delbert y Neil Salkind (2002), Handbook of research design \& social measurement, Sage, California, Thousand Oaks.

Ogden, Stuart y Robert Watson (1999), "Corporate performance and stakeholder management: balancing shareholder and customer interest in the U. K. privatized water industry", The Academy of Management Journal, 42(5) Academy of Management, Ada, Ohio, Estados Unidos, pp. 526-538.

Padua, Jorge, Ingvar Ahman, Héctor Apezechea y Carlos Borsotti (2001), Técnicas de investigación aplicadas a las ciencias sociales, México, El Colegio de México-Fondo de Cultura Económica.

Pearce, David y R. Kerry Turner (1990), Economics of natural resources and the environment, Baltimore, The Johns Hopkins University Press.

Peinado, Estrella (2004), Corporate social responsibility in Latin America and the Caribbean, Washington, D. C., InterAmerican Development Bank-Sustainable Development Department.

Salazar, Sabrina (2004), "La responsabilidad social corporativa en México como una herramienta estratégica para la competitividad: un enfoque econométrico y prospectivo", tesis de maestría, México, Instituto Tecnológico y de Estudios Superiores de Monterrey.

Salazar-Cantú, José de Jesús y Bryan Husted-Corregan (2005), La estrategia de responsabilidad social de la empresa a la luz de la teoría de juegos, México, Instituto Tecnológico y de Estudios Superiores de Monterrey.

Vanclay, Frank (2003), "Conceptual and methodological advances in social impact assessment", en H. Becker y F. Vanclay (eds.), The international handbook of social impact assessment, Northampton, Massachussets, Edward Elgar, pp. 1-9.

Waddock, Sandra y Samuel Graves (1997), "The corporate social performance-financial performance link”, Strategic 
Management Journal, 18(4), John Wiley \& Sons, Hoboken, Nueva Jersey, Estados Unidos, pp. 303-319.

Recibido: 30 de octubre de 2006. Reenviado: 26 de enero de 2007. Aceptado: 25 de abril de 2007.

José de Jesús Salazar Cantú. Es doctor en ciencias sociales con orientación en desarrollo sustentable por el Instituto de Investigaciones Sociales de la Universidad Autónoma de Nuevo León (UANL); asimismo realizó estudios de licenciatura en economía y maestría en ciencias de la administración en el Instituto Tecnológico y de Estudios Superiores de Monterrey, así como maestría en economía en la Facultad de Economía de la UANL. Sus líneas de investigación actuales son: efectos de la apertura comercial y responsabilidad social de la empresa. Entre sus publicaciones destacan: con Bryan Husted, "Taking Friedman seriously: Maximizing profits and social performance", Journal of Management Studies, XLIII(1), pp. 75-91 (2006); con André Varella, "Les effets des exportations sur la croissance du produit manufacturier dans les États mexicains", en F. Pinot (ed.), La mondialisation et ses effets: nouveaux débats, approches d'Europe et d'Amérique Latine, París, Universidad de París viI, pp. 71-80 (2006); con Bryan Husted, "Un estudio sobre la estrategia social de las empresas grandes ubicadas en México", Contaduría y Administración, 215, México, pp. 9-23 (2005).

Esthela Gutiérrez Garza. Es doctora en economía política por la Universidad de París viII. Realizó estudios de licenciatura en sociología en la Universidad de Monterrey y de maestría en estudios latinoamericanos en la Facultad de Ciencias Políticas y Sociales de la Universidad Nacional Autónoma de México. Actualmente es directora del Instituto de Investigaciones Sociales de la Universidad Autónoma de Nuevo León. Es miembro del Sistema Nacional de Investigadores, Nivel II. Es fundadora y directora de la revista Trayectorias. Dentro de sus actividades académicas destacan la coordinación de la colección en cuatro volúmenes de Testimonio de la crisis a finales de la década de los ochenta y la colección en cinco volúmenes de El debate nacional a finales de los años noventa. En 2006 elaboró, junto con un grupo de investigadores, el libro México. Diálogo social para un 
proyecto de nación, México, Instituto de Investigaciones Sociales de la Universidad Autónoma de Nuevo León. Ha publicado más de 80 artículos en revistas especializadas con arbitraje en México y en el extranjero. Su publicación más reciente es Teoría del desarrollo en América Latina, México, Trillas, 2003.

Karim Acuña Askar. Es doctor en ciencias con especialidad en salud ambiental por la Universidad de Tulane, en Nueva Orleáns. Sus investigaciones están orientadas a la generación de procesos tecnológicos que estimulen el desarrollo sustentable apoyando tanto la prevención de impacto ambiental como la restauración ambiental de los recursos naturales. Es profesor titular de la Universidad Autónoma de Nuevo León (UANL) adscrito a la Facultad de Medicina y colabora en el Instituto de Investigaciones Sociales. Es responsable del Laboratorio de Biorremediación Ambiental y de la Oficina de Procesamiento de Datos Ambientales de la UANL. Es miembro del Sistema Nacional de Investigadores, nivel I, así como de la International Water Association, Water Environment Federation, New York Academy of Sciences, American Chemical Society, American Society for Microbiology y la Sociedad Química de México. Obtuvo el Premio de Investigación UANL 2006 en Ciencias Exactas. Sus publicaciones más recientes son: en coautoría, "Effect of soil and a nonionic surfactant on BTE-OX and MTBE biodegradation kinetics", Water Science and Techonology, 52(8), pp. 107-115 (2005); en coautoría, "The Effect of a Nonionic Surfactant on the Biodegradation Kinetics of MTBE and BTEX", en B. M. Sass (ed.), Remediation of Chlorinated and Recalcitrant Compounds, Columbus, Ohio, Battelle Press, p. G54 (2006); en coautoría, "Biodegradation Kinetics of BTE-OX and MTBE by a diesel-grown biomass", Water Science and Technology, 53(11), Pergamon Press, Nueva York, pp. 197-204 (2006); en coautoría, "Transport of coliphage PRD1 in a surface flow constructed wetland", Water Environment Research, 78(11), Water Environment Federation, Alexandria, VA. EE.UU., pp. 2253-2260 (2006).

Bryan Husted Corregan. Es doctor por la Escuela Haas de Administración de Empresas de la Universidad de California, en Berkeley; asimismo realizó estudios de licenciatura en economía y maestría en administración en la Universidad Brigham Young. Actualmente es investigador nivel II en el Sistema Nacional de Investigadores (SNI) y es profesor de administración en la Escuela de 
Graduados en Administración y Dirección de Empresas (EGADE) del Instituto Tecnológico y de Estudios Superiores de Monterrey. Sus líneas de investigación actuales son: responsabilidad social y ambiental de la empresa. Entre sus publicaciones destacan: con David B. Allen, "Agency, information and the structure of moral problems in business", Organization Studies, XXVIII(2), W. de Gruyter, Nueva York, pp. 177-195 (2007); “Corporate social responsibility in the multinational enterprise: Strategic and institutional approaches", Journal of International Business Studies, XXXVII(6), Georgia State University, Atlanta, Ga., pp. 838-349 (2006); con José de Jesús Salazar Cantú, "Taking Friedman seriously: Maximizing profits and social performance", Journal of Management Studies, XLII(1), Blackwell Publishing en Durham, Reino Unido, pp. 75-91 (2006). 